\title{
Constitutive Modeling of Shape Memory Alloy Including Martensite-transformation-induced Plasticity
}

\author{
Tadashige Ikeda* \\ Department of Astronautics and Aeronautics, Chubu University, \\ 1200 Matsumoto-cho, Kasugai-shi, Aichi 487-8501, Japan
}

(Received March 27, 2020; accepted July 7, 2020)

Keywords: constitutive model, shape memory alloy, cyclic deformation, phase transformation, martensite-transformation-induced plasticity

The stress-strain curves of as-received shape memory alloys (SMAs), which are often used as sensors or/and actuators, vary among cyclic loadings. This phenomenon is caused by an irreversible process induced by the martensite transformation. To reproduce it mathematically, the one-dimensional phase transformation model previously proposed by the present author and his coworkers is developed. Specifically, new phases composed of the austenitic phase and the residual martensitic phase are introduced. To verify the validity of the model, cyclic tensile loading tests of as-received superelastic SMA wires are carried out and the measured stress-strain curves are compared with those obtained by calculation. The result shows that the present model can reproduce the variation of the stress-strain curve for the first several cycles with reasonable accuracy.

\section{Introduction}

Since shape memory alloys (SMAs) provide unique properties such as the shape memory effect and superelasticity, they have been applied to commercial products and studied to develop new applications in a wide range of fields such as aerospace, medicine, and home electronics. $^{(1,2)}$ In the applications, SMAs play the role of sensors or/and actuators as well as structural elements. For example, a water temperature regulator in a shower faucet comprises an SMA spring, a bias stainless spring, a knob compressing the bias spring for temperature adjustment, and inlet valves for hot and cold water. The length and stiffness of the SMA spring change with the water temperature. Accordingly, the inlet valves move so that the mixed water temperature approaches a set temperature. Although SMAs are or will be applied to many products, their deformation behaviors are complicated since such properties are based on a phase transformation and are nonlinear, showing hysteresis. To understand the mechanism of the complicated deformation behaviors and to design optimized products including SMAs, fundamental experimental data and mathematical models are necessary and many experimental and theoretical studies have already been carried out. ${ }^{(1-13)}$ From experiments, it is well known that the properties of as-received SMAs vary with increasing number of

*Corresponding author: e-mail: ikeda@isc.chubu.ac.jp

https://doi.org/10.18494/SAM.2020.2887 
loading cycles because stress-induced martensitic phases resulting from a transformation during the loading process cannot completely reverse-transform into the austenitic phase after unloading. The irreversibility of the process is due to the accumulation of dislocations, mismatches of grain boundaries, and other factors during the phase transformation. This martensite-transformation-induced plasticity (MTRIP) is one of the most important phenomena in the application of SMAs to products, because SMAs are usually used under cyclic loading in applications. ${ }^{(8-10)}$

Several types of constitutive models have been proposed in previous studies. ${ }^{(11-13)}$ However, most of them cannot describe the effects of MTRIP. To incorporate such effects into the constitutive models, Tanaka and coworkers assumed that the deformation behaviors were governed by macroscopic constitutive equations that did not include MTRIP, although local values for the stress, strain, and volume fraction of the martensitic phase were substituted into the equations. ${ }^{(14,15)}$ The local values included the effects of the residual stress, the residual strain, and the volume fraction of the residual martensitic phase. The residual stress was assumed to be an exponential function of time while the transformations took place and approached a limiting value with increasing time. The evolutions of the residual strain and the volume fraction were assumed to be functions of the residual stress. Lexcellent and Bourbon introduced the instantaneous fraction of the residual martensitic phase as a variable, which was assumed to be a function of the martensitic fraction accumulated only during the martensite transformation and to approach a limiting value. ${ }^{(16)}$ The specific free energy was assumed to vary with the instantaneous residual martensitic fraction, and accordingly, the transformation stresses also varied. Abeyaratne and Kim assumed that the residual stress at some location in a specimen increases with the number of times that the location has undergone each transformation. ${ }^{(17)}$ Bo and Lagoudas introduced the accumulated volume fraction of the detwinned martensite. ${ }^{(18)}$ The plastic strain was assumed to be a function of the accumulated detwinned martensitic volume fraction. The back stress was assumed to be a function of the plastic stress and the detwinned martensitic volume fraction, and the drag stress was assumed to be a function of the total martensitic volume fraction and the accumulated total martensitic volume fraction. Naito et al. introduced the residual martensitic phase connected in parallel to the austenitic phase in their one-dimensional model to consider the residual stress in the austenitic phase. $^{(19)}$ The volume fraction of the residual martensitic phase relative to the austenitic phase was assumed to increase with the number of loading cycles. Auricchio et al. assumed that the martensitic transformation stress decreased and the volume fraction of the residual martensitic phase increased with the accumulated martensitic volume fraction during the martensitic transformation and that the reverse transformation temperature increased with the accumulated martensitic volume fraction during the reverse transformation, and they approached limiting values. ${ }^{(20)}$

These models could closely reproduce the deformation behaviors of as-received SMAs under cyclic loadings with a constant strain amplitude or constant temperature amplitude by adequately determining the material constants related to the cyclic effect. However, these models except for Abeyaratne and Kim's model ${ }^{(17)}$ could not reasonably reproduce the deformation behaviors under cyclic loadings with varying amplitude. Abeyaratne and Kim 
reproduced such behaviors by considering a bar with a movable phase boundary between two phases, although this approach cannot reproduce the deformation behaviors of an SMA including more than two mixed phases.

The present author and his coworkers ${ }^{(21-25)}$ have also proposed constitutive models with an energy-based transformation criterion. To understand the physical background of the nonlinear deformation during the phase transformation, a grain-based micromechanical constitutive model was first presented. ${ }^{(21)}$ By increasing the number of grains to infinity in the micromechanical model, a lumped parameter model, referred to as the one-dimensional phase transformation model, was obtained. ${ }^{(22)}$ Moreover, this model was extended so as to be able to describe tension-compression asymmetric behavior and tension-torsion behavior. ${ }^{(23-25)}$ The features of this model are as follows: (1) more than two phases/variants can be taken into account, (2) the rate-dependent effect can be described, and (3) it can be used for quantitative analysis. However, this model could not reproduce the cyclic effects including MTRIP.

In this paper, this one-dimensional phase transformation model that can treat more than two phases and be used for quantitative analysis is extended so as to be able to describe cyclic effects including MTRIP. In Sect. 2, the features of the variation of the stress-strain curve under cyclic loadings are observed. The one-dimensional phase transformation model is explained and extended in Sect. 3. The validity of the extended model is shown by comparing the deformation behavior reproduced by the model with measured results in Sect. 4. In Appendix, a transformation criterion and a constitutive equation are derived.

\section{Experiment}

Figures 1(a) and 1(b) show quasistatic stress-strain curves of an as-received TiNi-SMA wire of $0.75 \mathrm{~mm}$ diameter (Kantoc, material code EF3256). This cyclic test was performed with a fatigue test machine (Shimadzu EHF-FB10kN-10LA). In Fig. 1(a), the wire was stretched

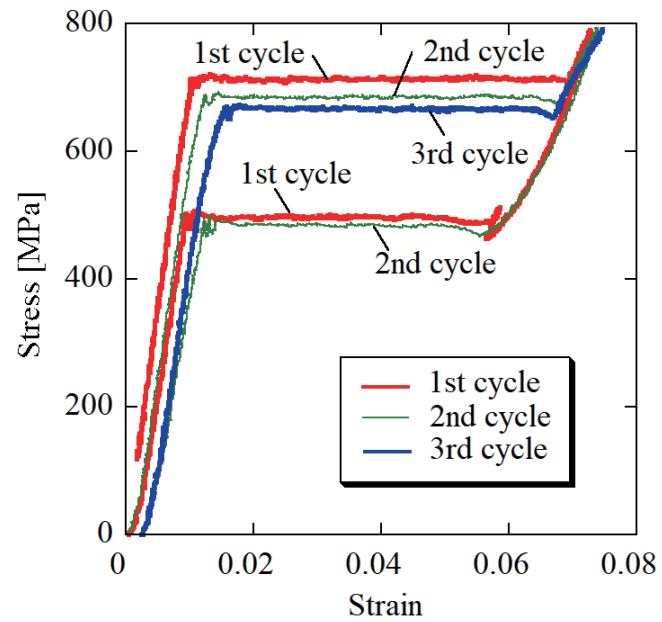

(a)

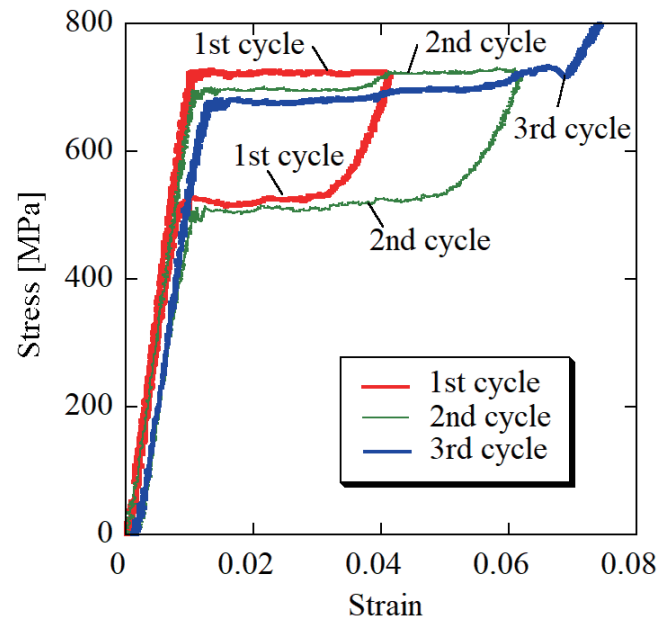

(b)

Fig. 1. (Color online) (a) Complete transformation cycles and (b) strain cycles with increasing return strain in stress-strain curves for the first few strain cycles of an as-received SMA wire. 
until the martensitic transformation was completed for all cycles. In Fig. 1(b), the return point of strain was $4 \%$ in the first cycle and $6 \%$ in the second cycle, and the transformation was completed in the third cycle. Hereafter, the return point of strain during the cyclic loading and unloading process at which the sign of the strain rate changes from positive to negative is referred to as the return strain.

It is seen from Figs. 1(a) and 1(b) that the features of the stress-strain curves during the first few cycles are as follows: With increasing number of cycles, (1) the martensitic transformation stress decreases, (2) the area of the hysteresis loop decreases, and (3) the residual strain increases. In addition, (4) as the return strain gradually increases during the cyclic loadings, the martensitic transformation stress increases in steps to the past return strains in the stress-strain curve.

\section{Constitutive Model}

Figure 2 shows a schematic of the stress-strain relation for the first few cycles of tensile loading and unloading, where the lattices represent schematics of crystal structures at several states. The residual martensitic phase is considered in addition to the austenitic phase and the martensitic phase. During the first loading (State 0 to State 3), the austenitic phase transforms into the martensitic phase when the stress increases beyond the martensitic transformation stress. However, during the first unloading (State 3 to State 5), not all the martensitic phase can return to the austenitic phase and some of the martensitic phase remains due to dislocation or mismatch between grains. During the subsequent second loading (State 5 to State 7), the returned austenitic phase transforms into the martensitic phase again. The transformation stress for the second loading is lower than that of the first loading owing to the residual stress generated by the residual martensitic phase inside the austenitic phase. Then, during the second unloading (State 7 to State 8), more martensitic phase remains and the residual strain increases, although the increment of the residual strain decreases.

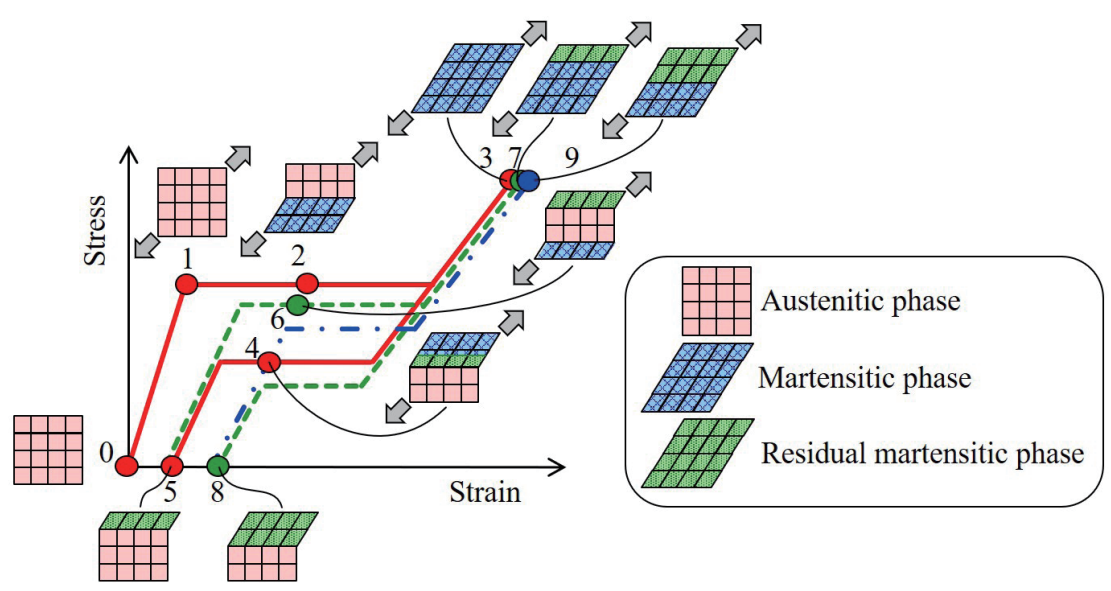

Fig. 2. (Color online) Variation of stress-strain curves for the first few strain cycles and schematics of corresponding crystal structures at the indicated states. 
To include the effect of the residual martensitic phase, mixed phases comprising the austenitic phase and the residual martensitic phase generated in the first cycle, the second cycle, and the $i$ th cycle are introduced, which are referred to as the $\mathrm{AR}_{1^{-}}, \mathrm{AR}_{2^{-}}$, and $\mathrm{AR}_{i}$-phases, respectively. Table 1 shows the correspondence between the crystal structures shown in Fig. 2 and those of the present model. The mixed phase in State 5 is assumed to be the $\mathrm{AR}_{1}$-phase and the mixed phase in State 8 is assumed to be the $A R_{2}$-phase, where the $A R_{2}$-phase has more of the residual martensitic phase than the $\mathrm{AR}_{1}$-phase. The correspondences between the crystal structures shown in Fig. 2 and those of the present model in States 4, 6, 7, and 9 are also listed in Table 1. Regarding the properties of the $\mathrm{AR}_{i}$-phase, the residual strain of the $\mathrm{AR}_{i}$-phase increases and the transformation stress from the austenitic phase to the $\mathrm{AR}_{i}$-phase decreases as the number of cycles $i$ of the $\mathrm{AR}_{i}$-phase increases.

Figure 3 shows the transformation rule of the one-dimensional phase transformation model for the present model including the $\mathrm{AR}_{i}$-phases. Here, an SMA bar specimen is considered and the specimen is assumed to consist of the austenitic phase represented by symbol $\mathrm{A}$, the martensitic phase represented by symbol $\mathrm{M}$, and the mixed phases represented by symbols $\mathrm{AR}_{1}$ and $A_{2}$. The required transformation energy (RTE) is defined as the energy required for the phase transformation caused by the interaction between grains or phases. It is assumed that

Table 1

(Color online) Relationship between schematic crystal structures in Fig. 2 and those in the present model.

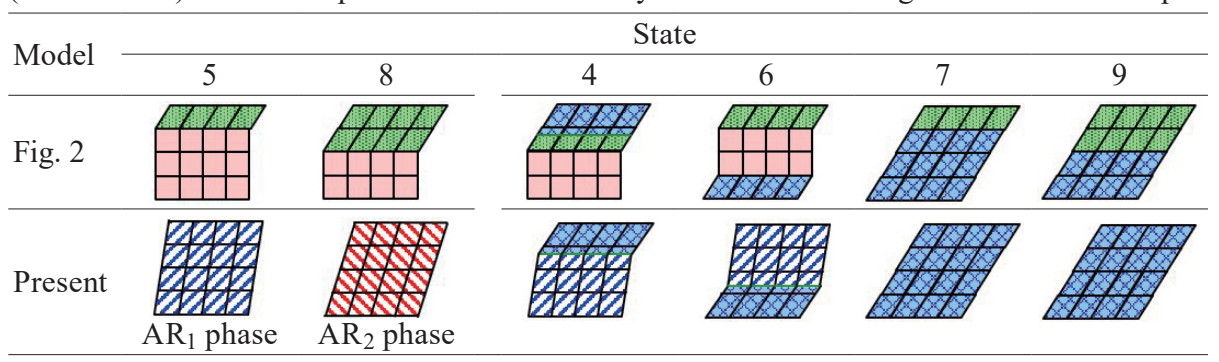

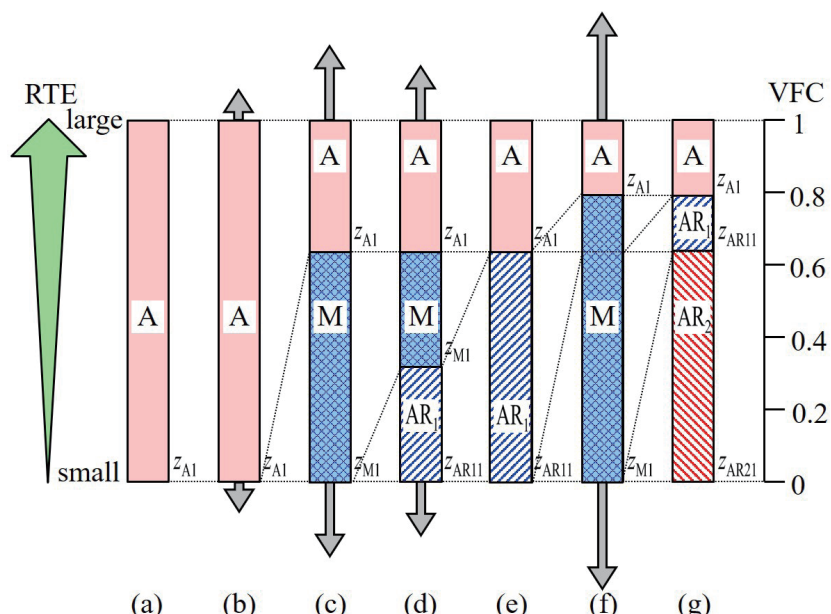

$\begin{array}{llllll}\text { (a) } & \text { (b) } & \text { (c) } & \text { (d) } & \text { (e) } & \text { (f) }\end{array}$

Fig. 3. (Color online) Phase transformation rule of the one-dimensional phase transformation model. 
infinitesimal virtual grains are sorted in order of RTE, so that RTE takes the minimum value at the bottom of the bar and the maximum value at the top. The order of these grains is assumed to be unchanged irrespective of the phases before and after the transformation. Accordingly, each phase transformation proceeds from the lower part to the upper part of the bar.

First, the whole specimen is assumed to be in the A-phase [Fig. 3(a)]. When a tensile force is applied to the bar and the stress value increases beyond a certain critical value, a transformation from the A-phase to the M-phase takes place from the bottom, at which the RTE takes the minimum value [Figs. 3(b) and 3(c)]. Then, when the tensile force is unloaded, the transformation from the M-phase to the $\mathrm{AR}_{1}$-phase takes place from the bottom [Fig. 3(d)]. Next, when the tensile force is applied again after the force is completely unloaded [Fig. 3(e)], the transformation from the $\mathrm{AR}_{1}$-phase to the $\mathrm{M}$-phase and then the transformation from the A-phase to the M-phase take place at the lowest boundary of the RTE for each transformation [Fig. 3(f)]. Finally, when the tensile force is unloaded, the $\mathrm{M}$-phase transformed from the $\mathrm{AR}_{1}$-phase transforms to the $\mathrm{AR}_{2}$-phase, and the $\mathrm{M}$-phase that has not yet transformed to the $\mathrm{AR}_{1}$-phase transforms to the $\mathrm{AR}_{1}$-phase. The transformations occur in the sequence of A-phase, M-phase, $\mathrm{AR}_{1}$-phase, M-phase, $\mathrm{AR}_{2}$-phase, M-phase, and so on.

When the length of the bar is normalized, the sum of the normalized regions in the same phase corresponds to the volume fraction of the phase. Hence, the ordinate is referred to as the volume fraction coordinate (VFC).

According to this assumption, the transformation criterion from the $\alpha$-phase to the $\beta$-phase is formulated as (see Appendix) ${ }^{(22-25)}$

$$
\frac{1}{2} \sigma^{2}\left(\frac{1}{E_{\beta}}-\frac{1}{E_{\alpha}}\right)+\sigma\left(\varepsilon_{\beta}-\varepsilon_{\alpha}\right)+\left(s_{\beta}-s_{\alpha}\right)\left(T-T_{\alpha \leftrightarrow \beta}\right)=\Psi_{\alpha \rightarrow \beta}\left[z_{\alpha 1}\right] .
$$

$\sigma, E_{\alpha}, \varepsilon_{\alpha}, s_{\alpha}, T$, and $T_{\alpha \leftrightarrow \beta}$ denote the stress, the Young's modulus of the $\alpha$-phase, the intrinsic strain due to the difference in the crystal structure between the $\alpha$-phase and a reference phase, the entropy of the $\alpha$-phase, the material temperature, and the ideal transformation temperature between the $\alpha$-phase and $\beta$-phase without dissipation due to the internal friction, respectively. $\Psi_{\alpha \rightarrow \beta}$ is the RTE for the transformation from the $\alpha$-phase to the $\beta$-phase, and $z_{\alpha 1}$ denotes the minimum value of the VFC in the $\alpha$-phase as shown in Fig. 3. The transformation criterion means that during the transformation process, the thermomechanical driving energy of the lefthand side is equal to the RTE. The relationship between $\Psi_{\alpha \rightarrow \beta}$ and $z_{\alpha 1}$ was found empirically to be approximated by the following formula:

$$
\Psi_{\alpha \rightarrow \beta}\left[z_{\alpha 1}\right]=\Psi_{c 1, \alpha \rightarrow \beta}+\Psi_{c 2, \alpha \rightarrow \beta}\left[1-a_{1, \alpha \rightarrow \beta}{ }^{-z_{\alpha 1}}+b_{\alpha \rightarrow \beta} a_{2, \alpha \rightarrow \beta}{ }^{-\left(1-z_{\alpha 1}\right)}\right]
$$

where $\Psi_{c 1, \alpha \rightarrow \beta}, \Psi_{c 2, \alpha \rightarrow \beta}, a_{1, \alpha \rightarrow \beta}, b_{\alpha \rightarrow \beta}$, and $a_{2, \alpha \rightarrow \beta}$ are material constants.

In this paper, an isothermal condition is considered. The strain under an isothermal condition is assumed to be the sum of the elastic strain and the intrinsic strain: 


$$
\varepsilon=\sigma \sum_{\alpha} \frac{z_{\alpha}}{E_{\alpha}}+\sum_{\alpha} \varepsilon_{\alpha} z_{\alpha}
$$

where $\varepsilon$ and $z_{\alpha}$ denote the strain and the volume fraction of the $\alpha$-phase, respectively.

\section{Results and Discussion}

\subsection{Validation of the proposed model}

To show the validity of the present model, the stress-strain curves shown in Figs. 1(a) and 1(b) are reproduced. An isothermal condition is assumed. The material and environmental constants are listed in Table 2.

Figures 4(a) and 4(b) show a comparison of the variation of the stress-strain curves for the first few strain cycles between the experiment and calculation. Symbols represent measured

Table 2

Material and environmental constants.

\begin{tabular}{|c|c|c|c|c|c|}
\hline$\alpha$ & \multicolumn{2}{|c|}{$E_{\alpha}[\mathrm{GPa}]$} & $\varepsilon_{\alpha}$ & \multicolumn{2}{|c|}{$\left(s_{M}-s_{\alpha}\right)\left(T-T_{\alpha \leftrightarrow M}\right)\left[\mathrm{MJ} / \mathrm{m}^{3}\right]$} \\
\hline A & \multicolumn{2}{|c|}{71} & 0 & \multicolumn{2}{|c|}{-31.5} \\
\hline M & \multicolumn{2}{|c|}{25} & 0.043 & \multicolumn{2}{|c|}{ - } \\
\hline $\mathrm{AR}_{1}$ & \multicolumn{2}{|c|}{67} & 0.001 & \multirow{2}{*}{\multicolumn{2}{|c|}{$\begin{array}{l}-29.35 \\
-271\end{array}$}} \\
\hline $\mathrm{AR}_{2}$ & \multicolumn{2}{|c|}{63} & 0.003 & & \\
\hline$\alpha \rightarrow \beta$ & $\begin{array}{c}\Psi_{c 1, \alpha \rightarrow \beta} \rightarrow \\
{\left[\mathrm{MJ} / \mathrm{m}^{3}\right]}\end{array}$ & $\begin{array}{c}\Psi_{c 2, \alpha \rightarrow \beta} \rightarrow \\
{\left[\mathrm{MJ} / \mathrm{m}^{3}\right]}\end{array}$ & $a_{1, \alpha \rightarrow \beta}$ & $a_{2, \alpha \rightarrow \beta}$ & $b_{\alpha \rightarrow \beta}$ \\
\hline $\mathrm{A} \rightarrow \mathrm{M}$ & 0 & 6.0 & $1.0 \times 10^{50}$ & $1.0 \times 10^{50}$ & 1.0 \\
\hline $\mathrm{M} \rightarrow \mathrm{AR}_{1}$ & 0 & 5.2 & $1.0 \times 10^{20}$ & $1.0 \times 10^{50}$ & 1.0 \\
\hline $\mathrm{AR}_{1} \rightarrow \mathrm{M}$ & 0 & 5.5 & $1.0 \times 10^{50}$ & $1.0 \times 10^{50}$ & 1.0 \\
\hline $\mathrm{M} \rightarrow \mathrm{AR}_{2}$ & 0 & 4.8 & $1.0 \times 10^{20}$ & $1.0 \times 10^{50}$ & 1.0 \\
\hline $\mathrm{AR}_{2} \rightarrow \mathrm{M}$ & 0 & 5.0 & $1.0 \times 10^{30}$ & $1.0 \times 10^{50}$ & 1.0 \\
\hline
\end{tabular}

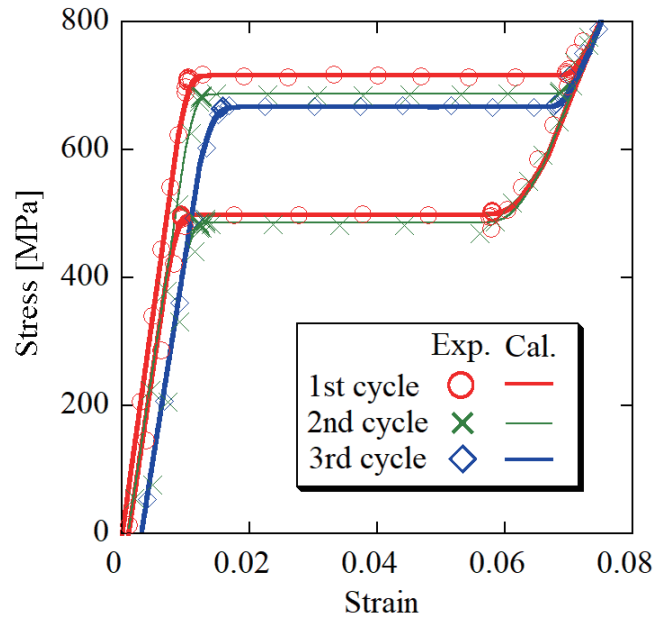

(a)

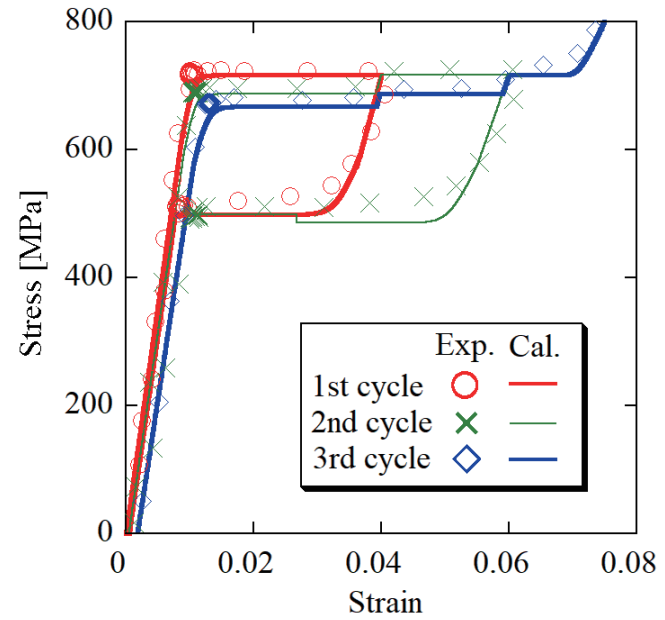

(b)

Fig. 4. (Color online) (a) Complete transformation cycles and (b) strain cycles with increasing return strain showing comparison of stress-strain curves for the first few strain cycles between experiment and calculation. 
data and lines are predictions. It is seen from Fig. 4(a) that the prediction can effectively capture the slopes of the elastic regions, the residual strain, and the reduction of the transformation stress by determining the material constants so that the predicted curves for the three cycles agree with the measured curves. This result shows that the constitutive equations are reasonable for describing the deformation behaviors including the phase transformation and the hysteresis. It is also seen from Fig. 4(b) that the prediction can capture the steplike increase in the transformation stress for the strain cycles with increasing return strain. However, the predicted reverse transformation stresses during the unloading are lower than those in the experiment. Accordingly, the assumed RTE is larger than the actual value. A reason for this difference is considered to be as follows. The energy required for the nucleation of the austenitic phase from the complete martensitic state is larger than that for expanding the austenitic region. The stressstrain curves during the unloading in Fig. 4(a) start with the nucleation of the austenitic phase, while those in Fig. 4(b) start with the development of the austenitic region. Since the material constants were determined so as to fit Fig. 4(a), the predicted reverse-transformation stresses are lower than those in the experiment in Fig. 4(b). The present model cannot distinguish this difference at the state when the transformation starts.

In this paper the evolution of the material constants for the mixed phases was not formulated and the material constants were given for each phase. A function that approaches a limiting value with increasing number of cycles can be adopted to the evolution function, such as an exponential function or an inverse proportional function. The form and values of coefficients of the function depend on the composition of the SMA and the thermomechanical treatment.

\subsection{Validation of RTE function}

The form of the RTE function is verified. From Eqs. (1) and (3), the formulae used to calculate the RTE and the volume fraction obtained from the experimental data under an isothermal condition can be obtained as

$$
\begin{gathered}
R T E_{A \rightarrow M}=\frac{1}{2} \sigma^{2}\left(\frac{1}{E_{M}}-\frac{1}{E_{A}}\right)+\sigma\left(\varepsilon_{M}-\varepsilon_{A}\right)+\left(s_{M}-s_{A}\right)\left(T-T_{A \leftrightarrow M}\right), \\
z_{M}=\frac{\left[\frac{\left(\varepsilon-\varepsilon_{A}\right)}{\sigma}-\frac{1}{E_{A}}\right]}{\left[\frac{\left(\varepsilon_{M}-\varepsilon_{A}\right)}{\sigma}+\left(\frac{1}{E_{M}}-\frac{1}{E_{A}}\right)\right]} .
\end{gathered}
$$

Open circles in Fig. 5 represent the martensite volume fraction-RTE relationship obtained from the first loading in Fig. 1(a). The RTE is 0 at the martensite volume fraction of 0 , increases steeply, then plateaus at approximately $6.0 \mathrm{MJ} / \mathrm{m}^{3}$, and finally increases steeply again. From this graph, the material constants in the RTE function shown in Eq. (2) are estimated. In this case, we can assume $\Psi_{c 1, A \rightarrow M}=0$ and $\Psi_{c 2, A \rightarrow M}=6.0 \mathrm{MJ} / \mathrm{m}^{3}$. Assuming $b_{A \rightarrow M}=1$, the combination 


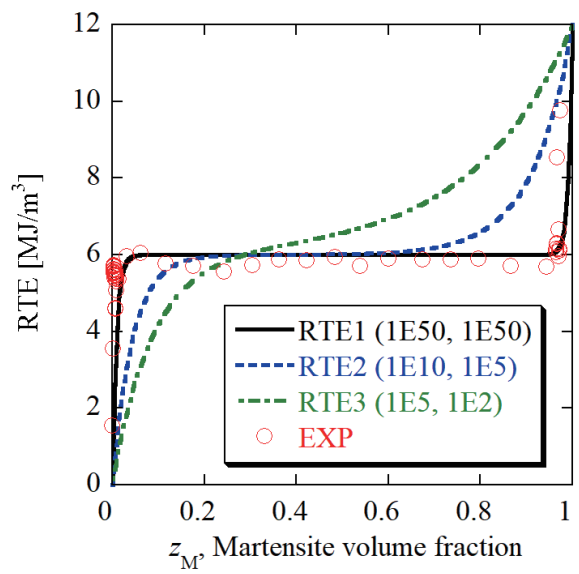

Fig. 5. (Color online) Validation of RTE function for $\left(\alpha_{1, A \rightarrow M}, \alpha_{2, A \rightarrow M}\right)$.

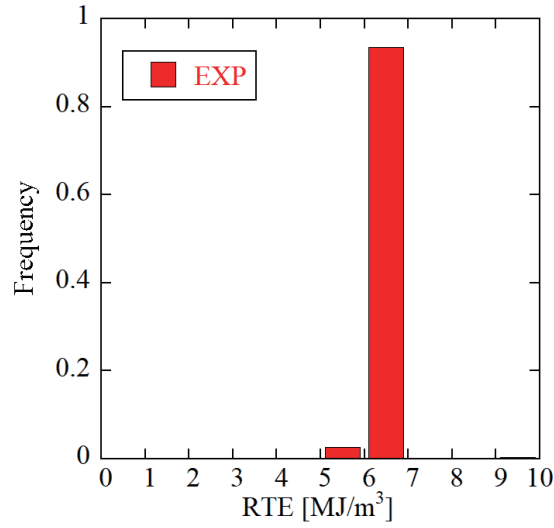

(a)

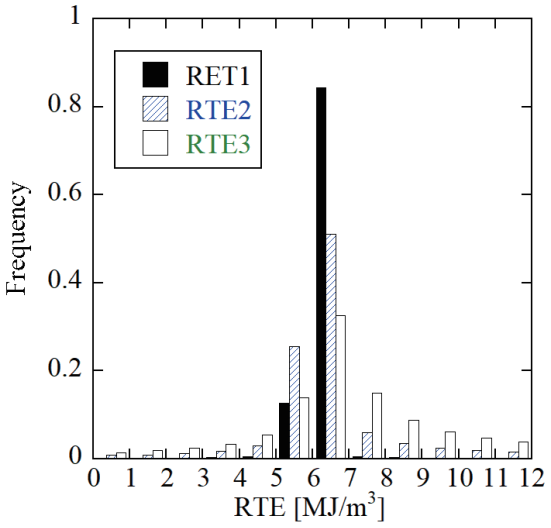

(b)

Fig. 6. (Color online) Frequency of RTE calculated from (a) the experimental data and (b) the example functions RTE1, RTE2, and RTE3.

of ( $\left.a_{1, A \rightarrow M}, a_{2, A \rightarrow M}\right)$ can be estimated easily through a trial-and-error approach. The curves in Fig. 5 are examples of approximation curves in the form of Eq. (2) having $\left(a_{1, A \rightarrow M}, a_{2, A \rightarrow M}\right)$ of $\left(10^{50}, 10^{50}\right),\left(10^{10}, 10^{5}\right)$, and $\left(10^{5}, 10^{2}\right) . \quad a_{1, A \rightarrow M}$ and $a_{2, A \rightarrow M}$ are related to the curvature of the left and right parts of the RTE curve, respectively. In this case, the measured data can be approximated best for $\left(a_{1, A \rightarrow M}, a_{2, A \rightarrow M}\right)=\left(10^{50}, 10^{50}\right)$. The inverse function of the RTE is a cumulative distribution function. The derivative of the cumulative distribution function is a probability density function. Figure 6 shows the discrete probability density function, that is, a histogram, with a bin width of $1 \mathrm{MJ} / \mathrm{m}^{3}$. It is seen that when larger values are given to $a_{1, A \rightarrow M}$ and $a_{2, A \rightarrow M}$, the kurtosis of the frequency increases.

\section{Conclusions}

The one-dimensional phase transformation model of SMAs, which are often used as sensors or/and actuators, proposed by the present author and his coworkers was extended so as to be 
able to describe cyclic deformation behaviors including the martensite-transformation-induced plasticity observed in experiments. The features of SMAs are as follows: with increasing number of cycles, (1) the martensitic transformation stress decreases, (2) the area of the hysteresis loop decreases, and (3) the residual strain increases. In addition, (4) the martensitic transformation stress increases in a steplike manner with increasing return strain during the cyclic loadings. To describe such features, mixed phases of the residual martensitic phase and austenitic phase were introduced into the model. A comparison of the predicted stress-strain curves with the measured data showed the validity of the model. However, the predicted curves are slightly different from those obtained by the experiment when the transformations are not completed, because the energy required to expand the region of a phase is assumed to be the same as that for the nucleation of the phase in the present model. The consideration of this difference in the model remains as a future challenge.

A three-dimensional finite element analysis of deformation behaviors of SMA elements could be performed, but its calculation would be time-consuming. Moreover, in most applications, the SMA elements are in the form of a wire, coil, or tube, so only one-directional movement need be considered. Therefore, the lumped parameter models in this paper are still useful, especially when calculations must be performed for many combinations of parameter values at an early stage of product design.

\section{Acknowledgments}

The author would like to thank Mr. Akira Nagai and Mr. Kazuya Sawamura, former graduate students at Nagoya University, for their support in the experiment.

\section{References}

1 K. Otsuka and C. M. Wayman, Eds.: Shape Memory Materials (Cambridge University Press, Cambridge, 1998).

2 K. Yamauchi, I. Ohkata, K. Tsuchiya, and S. Miyazaki, Eds.: Shape Memory and Superelastic Alloys: Applications and Technologies (Woodhead Publishing Limited, Cambridge, 2011).

3 J. A. Shaw, C. B. Churchill, and M. A. Iadicola: Exp. Tech. 32 (2008) 55. https://doi.org/10.1111/j.17471567.2008.00410.x

4 C. B. Churchill, J. A. Shaw, and M. A. Iadicola: Exp. Tech. 33 (2009) 51. https://doi.org/10.1111/j.17471567.2008.00460.x

5 C. B. Churchill, J. A. Shaw, and M. A. Iadicola: Exp. Tech. 33 (2009) 70. https://doi.org/10.1111/j.17471567.2009.00558.x

6 C. B. Churchill, J. A. Shaw, and M. A. Iadicola: Exp. Tech. 34 (2010) 63. https://doi.org/10.1111/j.17471567.2010.00619.x

7 B. Reedlunn, S. Daly, L. Hector Jr., P. Zavattieri, and J. Shaw: Exp. Tech. 37 (2013) 62. https://doi.org/10.1111/ j.1747-1567.2011.00717.x

8 S. Miyazaki, T. Imai, Y. Igo, and K. Otsuka: Metall. Trans. A 17 (1986) 115. https://doi.org/10.1007/ BF02644447

9 S. Miyazaki, Y. Igo, and K. Otsuka: Acta Metall. 34 (1986) 2045. https://doi.org/10.1016/0001-6160(86)902634

10 H. Tobushi, H. Iwanaga, K. Tanaka, T. Hori, and T. Sawada: Continuum Mech. Thermodyn. 3 (1991) 79. https://oi.org/10.1007/BF01129028 
11 L. G. Machado and D. C. Lagoudas: Shape Memory Alloys - Modeling and Engineering Applications, ed. D. C. Lagoudas (Springer Science+Business Media, LLC, New York, 2008) pp. 121-187. https://doi.org/10.1007/9780-387-47685-8 3

12 S. Barbarino, E. I. Saavedra Flores, R. M. Ajaj, I. Dayyani, and M. I. Friswell: Smart Mater. Struct. 23 (2014) 063001. https://doi.org/10.1088/0964-1726/23/6/063001

13 C. Cisse, W. Zaki, and T. B. Zineb: Smart Mater. Struct. 25 (2016) 103001. https://doi.org/10.1088/0964$1726 / 25 / 10 / 103001$

14 K. Tanaka, T. Hayashi, Y. Ito, and H. Tobushi: Mech. Mater. 13 (1992) 207. https://doi.org/10.1016/01676636(92)90003-V

15 K. Tanaka, F. Nishimura, T. Hayashi, H. Tobushi, and C. Lexcellent: Mech. Mater. 19 (1995) 281. https://doi. org/10.1016/0167-6636(94)00038-I

16 C. Lexcellent and G. Bourbon: Mech. Mater. 24 (1996) 59. https://doi.org/10.1016/0167-6636(96)00027-0

17 R. Abeyaratne and S.-J. Kim: Int. J. Solids Struct. 34 (1997) 3273. https://doi.org/10.1016/S00207683(96)00213-2

18 Z. Bo and D. C. Lagoudas: Int. J. Eng. Sci. 37 (1999) 1175. https://doi.org/10.1016/S0020-7225(98)00115-3

19 H. Naito, J. Sato, K. Funami, Y. Matsuzaki, and T. Ikeda: J. Intell. Mater. Syst. Struct. 12 (2001) 295. https:// doi.org/10.1106/1H1M-208B-7BHJ-67JD

20 F. Auricchio, S.Marfia, and E. Sacco: Comput. Struct. 81 (2003) 2301. https://doi.org/10.1016/S00457949(03)00319-5

21 F. A. Nae, Y. Matsuzaki, and T. Ikeda: Smart Mater. Struct. 12 (2003) 6. https://doi.org/10.1088/0964$1726 / 12 / 1 / 302$

22 T. Ikeda, F. A. Nae, H. Naito, and Y. Matsuzaki: Smart Mater. Struct. 13 (2004) 916. https://doi. org/10.1177/1045389X07077592

23 T. Ikeda: Proc. SPIE Smart Structures and Materials 2005, Ed. R. C. Smith 5757 (2005) 344. https://doi. org/10.1117/12.598693

24 T. Ikeda: J. Intell. Mater. Syst. Struct. 19 (2008) 533. https://doi.org/10.1177\%2F1045389X07077592

25 T. Ikeda: Proc. SPIE Smart Structures and Materials 2006, Ed. D. K. Lindner 6166 (2006) 61660Z. https://doi. org/10.1117/12.657779

\section{About the Author}

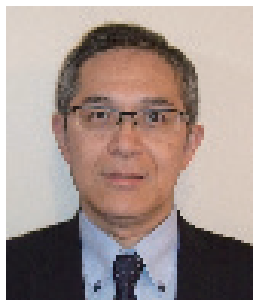

Tadashige Ikeda received his B.S., M.S., and Ph.D. degrees from Nagoya University, Japan, in 1988, 1990, and 1994, respectively. From 1993 to 1995, he was a technical official at Mechanical Engineering Laboratory, Japan. From 1995 to 2017, he was an assistant professor and then an associate professor at Nagoya University. Since 2017, he has been a professor at Chubu University. His research interests are in smart materials and structure systems, and composites. (ikeda@isc.chubu.ac.jp) 


\section{Appendix}

In this Appendix, Eq. (1) is derived briefly. From the first and second laws of thermodynamics, the Clausius-Duhem inequality can be obtained as

$$
\boldsymbol{\sigma}: \dot{\boldsymbol{\varepsilon}}+\rho(T \dot{s}-\dot{u})-\frac{\boldsymbol{q} \cdot \operatorname{grad} T}{T} \geq 0
$$

where $\sigma, \varepsilon, \rho, T, s, u$, and $\boldsymbol{q}$ denote the stress tensor, the strain tensor, the density of the material, the temperature, the entropy per unit mass, the internal energy per unit mass, and the heat flux transmitting across a unit area, respectively. The overdot denotes the time derivative. Assuming that the deformation is infinitesimal, the total strain can be given by a sum of the thermoelastic strain $\varepsilon^{e}$ and the transformation strain $\varepsilon^{t r}$ as

$$
\varepsilon=\varepsilon^{e}+\varepsilon^{t r}
$$

where the thermoelastic strain comprises the elastic strain and the thermal strain. The Gibbs free energy per unit mass is assumed to be given by a function of stress, temperature, and internal variables $\boldsymbol{Z}$ as

$$
G=G(\sigma, T, \boldsymbol{Z})=u-T s-\frac{1}{\rho} \sigma: \varepsilon^{e}
$$

Substituting Eqs. (A2) and (A3) into Eq. (A1), we have

$$
\boldsymbol{\sigma}: \dot{\boldsymbol{\varepsilon}}^{t r}-\left(\boldsymbol{\varepsilon}^{\boldsymbol{e}}+\rho \frac{\partial G}{\partial \boldsymbol{\sigma}}\right): \dot{\boldsymbol{\sigma}}-\left(s+\frac{\partial G}{\partial T}\right) \dot{T}-\left(\rho \frac{\partial G}{\partial \boldsymbol{Z}} \dot{\boldsymbol{Z}}+\frac{\boldsymbol{q} \cdot \operatorname{grad} T}{T}\right) \geq 0
$$

For thermoelastic deformation in a uniform temperature field, Eq. (A4) yields

$$
-\left(\boldsymbol{\varepsilon}^{e}+\rho \frac{\partial G}{\partial \boldsymbol{\sigma}}\right): \dot{\boldsymbol{\sigma}}-\left(s+\frac{\partial G}{\partial T}\right) \dot{T}=0
$$

Since Eq. (A5) must be satisfied for any $\dot{\sigma}$ and $\dot{T}$, we obtain the following thermoelastic constitutive equations and state equation:

$$
\begin{gathered}
\boldsymbol{\varepsilon}^{e}=-\rho \frac{\partial G}{\partial \boldsymbol{\sigma}}, \\
s=-\frac{\partial G}{\partial T} .
\end{gathered}
$$


Substituting Eqs. (A6) and (A7) into Eq. (A4), we obtain

$$
\boldsymbol{\sigma}: \dot{\boldsymbol{\varepsilon}}^{t r}-\left(\rho \frac{\partial G}{\partial \boldsymbol{Z}} \dot{\boldsymbol{Z}}+\frac{\boldsymbol{q} \cdot \operatorname{grad} T}{T}\right) \geq 0
$$

The internal variables $\boldsymbol{\varepsilon}^{t r}$ and $\boldsymbol{Z}$ can be obtained from their evolution equations.

A specific form of the Gibbs free energy is obtained as follows. Hooke's law of a homogeneous isotropic elastic body taking thermal expansion into account is given by

$$
\boldsymbol{\varepsilon}^{e}=-\frac{v}{E}(\operatorname{tr} \boldsymbol{\sigma}) \boldsymbol{I}+\frac{1+v}{E} \boldsymbol{\sigma}+\alpha_{T}\left(T-T_{r}\right) \boldsymbol{I},
$$

where $v, E, \boldsymbol{I}, \alpha_{T}$, and $T_{r}$ denote Poisson's ratio, the longitudinal elastic modulus, the identity tensor, the linear coefficient of thermal expansion, and the reference temperature, respectively. Substituting Eq. (A9) into Eq. (A6) and considering Eq. (A7) and $C_{p}=T(\partial s / \partial T)_{\sigma}$ for reversible deformation, we obtain

$$
\rho G=\frac{v}{2 E}(\operatorname{tr} \sigma)^{2}-\frac{1+v}{2 E} \sigma: \sigma-\alpha_{T}\left(T-T_{r}\right)(\operatorname{tr} \sigma)-\rho C_{p}\left[T \ln \frac{T}{T_{0}}-\left(T-T_{0}\right)\right]-\rho s_{0}\left(T-T_{0}\right),(\mathrm{A} 10)
$$

where $C_{p}$ is the specific heat per unit mass measured in the state of constant pressure, $T_{0}$ denotes the temperature at which the last two terms of Eq. (A10) vanish, and $s_{0}$ is the entropy per unit mass at $\sigma=0$ and $T=T_{0}$.

Here, a lumped parameter model is considered under unidirectional loading. In this case Eqs. (A2), (A6), (A7), (A8), and (A10) can respectively be written as

$$
\begin{gathered}
\varepsilon=\varepsilon^{e}+\varepsilon^{t r}, \\
\varepsilon^{e}=-\rho \frac{\partial G}{\partial \sigma}, \\
s=-\frac{\partial G}{\partial T}, \\
\sigma \dot{\varepsilon}^{t r}-\rho \frac{\partial G}{\partial \boldsymbol{Z}} \dot{\boldsymbol{Z}} \geq 0, \\
\rho G=-\frac{1}{2 E} \sigma^{2}-\alpha_{T}\left(T-T_{r}\right) \sigma-\rho C_{p}\left[T \ln \frac{T}{T_{0}}-\left(T-T_{0}\right)\right]-\rho s_{0}\left(T-T_{0}\right) .
\end{gathered}
$$


Since an SMA is composed of the austenitic phase and variants of the martensitic phase, the Gibbs free energy and entropy are assumed to be their sums for each phase.

$$
\begin{gathered}
G\left(\sigma, T, z_{\alpha}\right)=\sum_{\alpha} z_{\alpha} G_{\alpha}(\sigma, T) \\
s_{0}=\sum_{\alpha} z_{\alpha} s_{\alpha 0}
\end{gathered}
$$

$G_{\alpha}$ is the Gibbs free energy of the $\alpha$-phase and

$$
\rho G_{\alpha}=-\frac{1}{2 E_{\alpha}} \sigma^{2}-\alpha_{T}\left(T-T_{r}\right) \sigma-\rho C_{p}\left[T \ln \frac{T}{T_{0}}-\left(T-T_{0}\right)\right]-\rho s_{\alpha 0}\left(T-T_{0}\right)
$$

from Eq. (A15). $s_{\alpha 0}$ is the entropy of the $\alpha$-phase per unit mass at $T=T_{0}$ and $\sigma=0 . \quad z_{\alpha}$ is the internal variable referred to as the volume fraction of the $\alpha$-phase and satisfies the relation

$$
\sum_{\alpha} z_{\alpha}=1
$$

" $\alpha$ " is "A" for the austenite phase, " $\mathrm{M}$ " for the martensitic phase, etc. For simplicity, the linear coefficient of thermal expansion $\alpha_{T}$, the specific heat $C_{p}$, and the density $\rho$ are assumed to take the same values for any phase. The thermoelastic strain can be obtained as

$$
\varepsilon^{e}=\sigma \sum_{\alpha} \frac{z_{\alpha}}{E_{\alpha}}+\alpha_{T}\left(T-T_{r}\right)
$$

by substituting Eqs. (A16) and (A18) into Eq. (A12). The transformation strain $\varepsilon^{t r}$ is assumed to be given as

$$
\varepsilon^{t r}=\sum_{\alpha} z_{\alpha} \varepsilon_{\alpha}
$$

where $\varepsilon_{\alpha}$ is the intrinsic transformation strain of phase $\alpha$. From Eqs. (A11), (A20), and (A21), the constitutive equation (3) can be obtained. Substituting Eq. (A21) into Eq. (A14), we have

$$
\sum_{\alpha}\left(\sigma \varepsilon_{\alpha}-\rho \frac{\partial G}{\partial z_{\alpha}}\right) \dot{z}_{\alpha} \geq 0 .
$$

Although the phase transformation develops when Eq. (A22) is satisfied, more severe conditions than Eq. (A22) are adopted for a simpler formulation so that Eq. (A22) is satisfied for each phase transformation. Accordingly, we assume the phase transformation from the $\alpha$-phase to the $\beta$-phase proceeds when 


$$
\left[\sigma\left(\varepsilon_{\beta}-\varepsilon_{\alpha}\right)+\frac{1}{2}\left(\frac{1}{E_{\beta}}-\frac{1}{E_{\alpha}}\right) \sigma^{2}+\rho\left(s_{\beta 0}-s_{\alpha 0}\right)\left(T-T_{0}\right)\right] \dot{z}_{\alpha \rightarrow \beta} \geq 0,
$$

where $z_{\alpha \rightarrow \beta}$ is the volume fraction of the $\beta$-phase transformed from the $\alpha$-phase. Since $\dot{z}_{\alpha \rightarrow \beta} \geq 0$ for the phase transformation from the $\alpha$-phase to the $\beta$-phase, we obtain

$$
\sigma\left(\varepsilon_{\beta}-\varepsilon_{\alpha}\right)+\frac{1}{2}\left(\frac{1}{E_{\beta}}-\frac{1}{E_{\alpha}}\right) \sigma^{2}+\rho\left(s_{\beta 0}-s_{\alpha 0}\right)\left(T-T_{0}\right)=\Psi_{\alpha \rightarrow \beta},
$$

where $\Psi_{\alpha \rightarrow \beta} \geq 0$. This is the phase transformation criterion of Eq. (1). The left-hand side of Eq. (A24) is the driving force for the phase transformation from the $\alpha$-phase to the $\beta$-phase; $\Psi_{\alpha \rightarrow \beta}$ in the right-hand side is the RTE from the $\alpha$-phase to the $\beta$-phase. 